\title{
UNBOUNDED POINCARÉ DOMAINS
}

\author{
Ritva Hurri-Syrjänen \\ University of Jyväskylä, Department of Mathematics \\ P.O. Box 35, SF-40351 Jyväskylä, Finland
}

\begin{abstract}
We show that unbounded John domains $D$ in $\mathbf{R}^{n}$ satisfy the Poincaré inequality

$$
\inf _{a \in \mathbf{R}}\|u-a\|_{L^{q}(D)} \leq c\|\nabla u\|_{L^{p}(D)}
$$
\end{abstract}

where $q=n p /(n-p), 1 \leq p<n, c=c(p, q, D)$, and $u \in L^{p}(D)$, and that in a certain sense John domains form the largest subclass of $(n p /(n-p), p)$-Poincaré domains.

\section{Introduction}

A domain $D$ in $\mathbf{R}^{n}$ with finite $n$-Lebesgue measure $|D|$ is a $p$-Poincaré domain, if there is a constant $c=c(p, D)<\infty$ such that

$$
\left\|u-u_{D}\right\|_{L^{p}(D)} \leq c\|\nabla u\|_{L^{p}(D)}
$$

for all $u \in W_{p}^{1}(D), p \in[1, \infty)$; here $u_{D}$ is the average function of $u$ over $D$. A bounded domain with a smooth boundary was known to be a $p$-Poincaré domain for years. Recently, bounded Poincaré domains have been under very extensive study, see e.g. [EH], [H1], [M], [Maz] and [SS1-3]. Now it is well known that bounded John domains form a proper subclass of $p$-Poincaré domains for each $p \in[1, \infty)$.

However, not very much attention has been paid to unbounded domains with infinite $n$-Lebesgue measure. If the measure of $D$ is infinite, (1.1) should be replaced by

$$
\inf _{a \in \mathbf{R}}\|u-a\|_{L^{q}(D)} \leq c\|\nabla u\|_{L^{p}(D)}
$$

where $q=n p /(n-p), 1 \leq p<n, c=c(p, D)$, and $u \in L_{p}^{1}(D)$. We call domains $D$ satisfying $(1.2)(n p /(n-p), p)$-Poincaré domains and we write $D \in$ $\mathscr{P}(n p /(n-p), p)$.

1991 Mathematics Subject Classification: Primary 46E35; Secondary 26D10.

Part of this paper was done while the author was visiting The University of Hawaii and it was completed during her stay at The University of Texas at Austin. She wishes to thank both universities for their hospitality. 
In this paper we concentrate on the extremum case $q=n p /(n-p)$. Somewhat implicit characterizations for $(n p /(n-p), p)$-Poincaré domains have been given by V.G. Maz'ya. R. Andersson notices that the whole space $\mathbf{R}^{n}$ satisfies (1.2), [A]. B. Bojarski has shown that (1.2) is true for a bounded John domain.

We will prove that unbounded John domains are $(n p /(n-p), p)$-Poincaré domains for $1 \leq p<n$, Theorem 4.6. Our examples illuminate the fact that in a certain sense John domains form the largest subclass of $(n p /(n-p), p)$-Poincaré domains, Examples 3.5, 3.7, and 4.3.

We consider also weighted inequalities:

$$
\inf _{a \in \mathbf{R}}\|u-a\|_{L^{q}(D, w)} \leq c\|\nabla u\|_{L^{p}(D, w)}
$$

where $w$ is a weight function, $c$ is a constant which does not depend on a function $u$, and $1 \leq p \leq q \leq n p /(n-p)$.

Inequalities with different weights have been studied widely; see for example $[\mathrm{EO}],[\mathrm{FKS}],[\mathrm{GO}]$ and references therein, [H2], [IN], [K] and [Maz]. A related inequality to (1.3) is satisfied by a John domain, if $1 \leq p \leq q \leq k p$, and $k$ is slightly bigger than one, and $w$ is the so called Muckenhoupt weight; [FKS], [IN]. We show that John domains satisfy (1.3) with $q=n p /(n-p), 1<p<n$, when $w$ is an $A_{p, q}$-weight; that is, there is a constant $c<\infty$ such that

$$
\left(\frac{1}{|Q|} \int_{Q} w(x)^{q} d x\right)^{1 / q}\left(\frac{1}{|Q|} \int_{Q}(w(x))^{-p /(p-1)} d x\right)^{(p-1) / p} \leq c
$$

for all cubes $Q \subset \mathbf{R}^{n}$.

I wish to thank O. Martio and W. Smith for reading my manuscript and J. Väisälä for pointing out to me his Theorem 4.5.

\section{Notation}

Throughout this paper we let $D$ be a domain of euclidean $n$-space $\mathbf{R}^{n}, n \geq 2$. We suppose that $p \in[1, \infty)$ and $q \in[1, \infty)$ unless otherwise stated.

Let $C^{\infty}(D)$ denote the space of functions on $D$ which are infinitely differentiable on $D$. The space $L^{p}(D)$ is a set of Lebesgue measurable functions $u$ on $D$ for which

$$
\|u\|_{L^{p}(D)}=\left(\int_{D}|u(x)|^{p} d x\right)^{1 / p}<\infty .
$$

Let $L^{p}(D$, loc $)$ denote the space of functions which are locally integrable of order $p$ on $D$. The space of Lebesgue measurable functions on $D$ with the first distributional derivatives in $L^{p}(D)$ is denoted by $L_{p}^{1}(D)$. We equip $L_{p}^{1}(D)$ with the seminorm $\|\nabla u\|_{L^{p}(D)}$; here $\nabla u=\left(\partial_{1} u, \ldots, \partial_{n} u\right)$ is the gradient of $u$. The following properties of the space $L_{p}^{1}(D)$ are recalled: 
2.1. Lemma $\left[\mathrm{Maz}, 1.1 .2\right.$. Theorem]. Any element of $L_{p}^{1}(D)$ is in $L^{p}(D$, loc $)$.

2.2. Lemma $\left[\mathrm{Maz}, 1.1 .5\right.$. Theorem 1]. The space $L_{p}^{1}(D) \cap C^{\infty}(D)$ is dense in $L_{p}^{1}(D)$.

In the Sobolev space $W_{p}^{1}(D)=L^{p}(D) \cap L_{p}^{1}(D)$ we use the norm

$$
\|u\|_{W_{p}^{1}(D)}=\|u\|_{L^{p}(D)}+\|\nabla u\|_{L^{p}(D)} .
$$

The average of a function $u$ over a domain $A$ with finite Lebesgue measure $|A|$ is

$$
u_{A}=\frac{1}{|A|} \int_{A} u(x) d x .
$$

Definition. Let $D \subset \mathbf{R}^{n}$ be a domain and let $1 \leq p \leq q<\infty$. If there is a constant $c=c(p, q, D)<\infty$ such that

$$
\inf _{a \in \mathbf{R}}\|u-a\|_{L^{q}(D)} \leq c\|\nabla u\|_{L^{p}(D)}
$$

whenever $u \in L_{p}^{1}(D)$, then $D$ is a $(q, p)$-Poincaré domain and we write $D \in$ $\mathscr{P}(q, p)$.

Let $w$ be a non-negative measurable function. The space $L^{p}(D, w)$ is a set of functions $u$ on $D$ such that

$$
\|u\|_{L^{p}(D, w)}=\left(\int_{D}|u(x)|^{p} w(x)^{p} d x\right)^{1 / p}<\infty .
$$

The weighted Sobolev space $W_{p}^{1}(D, w)$ is the space of functions $u \in L^{p}(D, w)$, whose first distributional partial derivatives belong to $L^{p}(D, w)$. In $W_{p}^{1}(D, w)$ we use the norm

$$
\|u\|_{W_{p}^{1}(D, w)}=\|u\|_{L^{p}(D, w)}+\|\nabla u\|_{L^{p}(D, w)} .
$$

The weighted average of $u$ over $D$ is

$$
u_{D, w}=\left(\int_{D} w(x) d x\right)^{-1} \int_{D} u(x) w(x) d x
$$

where we suppose that $\int_{D} w(x) d x<\infty$.

Let $A$ be a set. The euclidean distance from $x \in A$ to the boundary of $A$ is written as $d(x, \partial A)$. We let $\operatorname{dia}(A)$ and $\bar{A}$ denote the diameter of $A$ and the closure of $A$, respectively.

We let $c(*, \cdots, *)$ denote a constant which depends only on the quantities appearing in the parentheses. 


\section{Domains with finite $n$-Lebesgue measure}

A domain $D$ is called an $(\alpha, \beta)$-John domain, $0<\alpha \leq \beta<\infty$, if there is $x_{0} \in D$ such that each $x \in D$ can be joined to $x_{0}$ by a curve $\gamma:[0, \ell] \rightarrow D$ parametrized by arc length with $\ell \leq \beta$ and

$$
d(\gamma(t), \partial D) \geq \frac{\alpha}{\ell} t, \quad t \in[0, \ell],
$$

[MS]. We write $D \in J(\alpha, \beta)$, and we say $x_{0}$ is a John center. An $(\alpha, \beta)$-John domain is bounded.

3.1. Theorem. Suppose that $D \in J(\alpha, \beta)$. Let $1 \leq p<n$ and $p \leq q \leq$ $n p /(n-p)$. Then

$$
\left\|u-u_{D}\right\|_{L^{q}(D)} \leq c\|\nabla u\|_{L^{p}(D)},
$$

where $c=c(n, p, q)(\beta / \alpha)|D|^{1 / n+1 / q-1 / p}$ and $u \in L_{p}^{1}(D)$.

Theorem 3.1 is proved by B. Bojarski, [B]. A careful calculation gives the above mentioned constant, see [H1, Lemma 8.3].

The quasihyperbolic distance between points $x_{1}$ and $x_{2}$ in $D$ is given by

$$
k_{D}\left(x_{1}, x_{2}\right)=\inf _{\gamma} \int_{\gamma} \frac{d s}{d(x, \partial D)}
$$

where the infimum is taken over all rectifiable curves $\gamma$ joining $x_{1}$ and $x_{2}$ in $D$, [GP].

A domain $D$ satisfies a quasihyperbolic boundary condition, if for $x_{0} \in D$ and for some $a>1$

$$
k_{D}\left(x_{0}, x\right) \leq a \log \left(1+\frac{\left|x_{0}-x\right|}{\min \left\{d\left(x_{0}, \partial D\right), d(x, \partial D)\right\}}\right)
$$

for all $x \in D$.

John domains form a proper subclass of domains satisfying a quasihyperbolic boundary condition, [GM, Lemma 3.11 and Remark 3.13].

A Whitney cube \#-condition. Suppose that $|D|<\infty$ and that

$$
D=\bigcup_{k=1}^{\infty} \bigcup_{j=1}^{N_{k}} Q_{j}^{k}
$$

where the Whitney decomposition of $D$, see [S, Chapter IV], is arranged so that for Whitney cubes $Q_{j}^{k}$

$$
\operatorname{dia}\left(Q_{j}^{k}\right)=|D|^{1 / n} 2^{-k} \quad \text { for } j=1, \ldots, N_{k} .
$$


We say that $D$ satisfies a Whitney cube \#-condition, if there are constants $M<$ $\infty$ and $\lambda \in(0, n)$ such that

$$
N_{k} \leq M 2^{\lambda k} \quad \text { for } k=1,2, \ldots
$$

[MV, Chapter 2].

Bounded domains satisfying a quasihyperbolic boundary condition are called Hölder domains by W. Smith and D. Stegenga. In fact, domains with finite Lebesgue measure satisfying a quasihyperbolic boundary condition are bounded. I wish to thank W. Smith for discussions concerning Theorem 3.3. For the proof we need the following lemma, see [H1, Lemma 7.23] for a bounded case.

3.2. Lemma. Suppose that $D$ with $|D|<\infty$ is a domain which satisfies a quasihyperbolic boundary condition such that for some $x_{0} \in D$ and $a>1$

$$
k_{D}\left(x_{0}, x\right) \leq a \log \left(1+\frac{\left|x_{0}-x\right|}{\min \left\{d\left(x_{0}, \partial D\right), d(x, \partial D)\right\}}\right)
$$

for all $x \in D$. Let $\gamma$ be a quasihyperbolic geodesic joining $x$ to $x_{0}$ in $D$. Then for each $y \in \gamma$

$$
l(\gamma(x, y)) \leq c_{1} d(y, \partial D)^{1 / 2 a}\left(\frac{|D|^{1 / n}+c_{0}\left|x_{0}-x\right|}{d\left(x_{0}, \partial D\right)^{1 / a}}\right)^{(2 a-1) /(2(a-1))}
$$

where $l(\gamma(x, y))$ is the length of $\gamma$ between points $x$ and $y$,

$$
c_{0}=\sup _{x \in D}(d(x, \partial D)) /\left(d\left(x_{0}, \partial D\right)\right), \quad \text { and } \quad c_{1}=c_{1}\left(a, c_{0}\right) .
$$

Proof. Since $|D|<\infty$,

$$
k_{D}\left(x_{0}, x\right) \leq a \log \left(1+\frac{\left|x_{0}-x\right|}{\min \left\{d\left(x_{0}, \partial D\right), d(x, \partial D)\right\}}\right) \leq a \log \left(1+\frac{c_{0}\left|x_{0}-x\right|}{d(x, \partial D)}\right)
$$

for all $x \in D$, where

$$
c_{0}=\sup _{x \in D} \frac{d(x, \partial D)}{d\left(x_{0}, \partial D\right)} \leq \frac{|D|^{1 / n}}{d\left(x_{0}, \partial D\right)}<\infty
$$

After this observation the proof is similar to the proof of [GHM, Lemma 3.1] and [H1, Lemma 7.23].

3.3. Theorem. If a domain $D$ satisfying a quasihyperbolic boundary condition has finite $n$-Lebesgue measure $|D|<\infty$, then $D$ is bounded. 
Proof. Suppose that $D \in \mathbf{R}^{n}$ with $|D|<\infty$ is a domain which satisfies a quasihyperbolic boundary condition such that for some $x_{0} \in D$ and $a>1$

$$
k_{D}\left(x_{0}, x\right) \leq a \log \left(1+\frac{\left|x_{0}-x\right|}{\min \left\{d\left(x_{0}, \partial D\right), d(x, \partial D)\right\}}\right)
$$

for all $x \in D$. Assume that $D$ is unbounded. We show that this leads to a contradiction. Fix $\varepsilon>0$. Denote $c_{0}=\sup _{x \in D}(d(x, \partial D)) /\left(d\left(x_{0}, \partial D\right)\right)$. Let $M>\left(|D|^{1 / n}\right) / 2 c_{0}$ such that $d(x, \partial D)<\varepsilon$ for all $x \in \mathbf{R}^{n} \backslash B\left(x_{0}, M\right)$. Let $x_{1} \in D \cap$ $S^{n-1}\left(x_{0}, 3 M\right)$ and fix a quasihyperbolic geodesic $\gamma\left(x_{0}, x_{1}\right)$. Pick $x_{2} \in \gamma\left(x_{0}, x_{1}\right)$ with $x_{2} \in D \cap S^{n-1}\left(x_{0}, 2 M\right)$ and $x_{3} \in \gamma\left(x_{0}, x_{1}\right)$ with $x_{3} \in D \cap S^{n-1}\left(x_{0}, M\right)$.

Since $d(x, \partial D)<\varepsilon$ for all $x \in \gamma\left(x_{2}, x_{3}\right)$,

$$
k_{D}\left(x_{2}, x_{3}\right)=\inf _{\gamma} \int_{\gamma} \frac{d s}{d(x, \partial D)} \geqq M / \varepsilon
$$

On the other hand Lemma 3.2 yields

$$
\begin{aligned}
k_{D}\left(x_{2}, x_{3}\right) & \leq k_{D}\left(x_{0}, x_{2}\right) \\
& \leq a \log \left(1+\frac{\left|x_{0}-x_{2}\right|}{\min \left\{d\left(x_{0}, \partial D\right), d\left(x_{2}, \partial D\right)\right\}}\right) \\
& \leq a \log \left(1+\frac{2 c_{0} M}{d\left(x_{2}, \partial D\right)}\right) \\
& \leq a \log c_{1}\left(\frac{|D|^{1 / n}+2 c_{0} M}{l\left(\gamma\left(x_{2}, x_{3}\right)\right)^{2 a}}\right)\left(\frac{|D|^{1 / n}+c_{0} M}{d\left(x_{0}, \partial D\right)^{1 / a}}\right)^{(2 a-1) /(2(a-1))} \\
& \leq c_{2} \log c_{3} M
\end{aligned}
$$

since $a>1$ and $|D|^{1 / n} \leq 2 c_{0} M$; here $c_{i}=c_{i}\left(a, c_{0}, d\left(x_{0}, \partial D\right),|D|\right), i=2,3$.

Now, if $\varepsilon$ goes to 0 , we obtain a contradiction. Theorem 3.3 is proved.

Our following theorem generalizes the result of [SS2, Corollary 8].

3.4. Theorem. Suppose that a domain $D$ with $|D|<\infty$ satisfies a quasihyperbolic boundary condition with a constant $a$.

If $p<n$, then

(i) there is a constant $p_{0}=p_{0}(D)<n$ such that $D \in \mathscr{P}(p, p)$ for $p>p_{0}$,

(ii) there is a constant $p_{1}=p_{1}(q, D)<n$ such that $D \in \mathscr{P}(q, p)$ for $p_{1}<p \leq$ $q<((n-\lambda) n p) /(a(n-p))$, where $\lambda<n$ is a Whitney cube \#-condition constant. 
Proof. Let $W$ be a Whitney decomposition of $D$. Fix $Q_{0} \in W$ with $x_{0} \in Q_{0}$. By [H1, Lemma 7.13] each cube $Q \in W$ can be joined to $Q_{0}$ by a chain of cubes $Q_{j} \in W, j=0,1, \ldots, s, Q_{s}=Q$, such that

$$
\left|Q_{\ell}\right|^{a} \leq c(n, D)\left|Q_{j}\right|, \quad \ell \geq j .
$$

Now

$$
\int_{D}\left|u(x)-u_{Q_{0}}\right|^{q} d x \leq 2^{q} \sum_{Q \in W} \int_{Q}\left|u(x)-u_{Q}\right|^{q} d x+2^{q} \sum_{Q \in W} \int_{Q}\left|u_{Q}-u_{Q_{0}}\right|^{q} d x
$$

where by Theorem 3.1

$$
\begin{aligned}
\sum_{Q \in W} \int_{Q}\left|u(x)-u_{Q}\right|^{q} d x & \leq c_{1}(n, p, q) \sum_{Q \in W}\left(|Q|^{1 / n+1 / q-1 / p}\right)^{q}\left(\int_{Q}|\nabla u(x)|^{p} d x\right)^{q / p} \\
& \leq c_{2}(n, p, q, D)\left(\int_{D}|\nabla u(x)|^{p} d x\right)^{q / p}
\end{aligned}
$$

since $p \leq q \leq(n p) /(n-p)$.

As in [H1, the proof for Theorem 4.4] we obtain using [H1, Proposition 6.1] and Theorem 3.1

$$
\begin{aligned}
\sum_{Q \in W} \int_{Q}\left|u_{Q}-u_{Q_{0}}\right|^{q} d x \leq & c_{3}(n, p, q) \sum_{Q \in W} \int_{Q} k_{D}\left(x_{0}, x\right)^{q-1} d x \times \\
& \times \sum_{\substack{j=0 \\
Q_{s}=Q}}^{s}\left(\left|Q_{j}\right|^{1 / n-1 / p}\left(\int_{Q_{j}}|\nabla u(x)|^{p} d x\right)^{1 / p}\right)^{q} .
\end{aligned}
$$

If $p<n$, then [H1, Lemma 7.13] and [SS3, Corollary 1] yield

$$
\begin{aligned}
& \sum_{Q \in W} \int_{Q}\left|u_{Q}-u_{Q_{0}}\right|^{q} d x \\
& \quad \leq c_{3}(n, p, q) \sum_{Q \in W} \int_{Q} k_{D}\left(x_{0}, x\right)^{q-1} d x|Q|^{q q(1 / n-1 / p)}\|\nabla u\|_{L^{p}(D)}^{q} \\
& \quad \leq c_{3}(n, p, q) \sum_{j=1}^{\infty} \sum_{\substack{Q \in W \\
\operatorname{dia}(Q)=|D|^{1 / n} 2^{-j}}} \int_{Q} k_{D}\left(x_{0}, x\right)^{q-1} d x|Q|^{a q(1 / n-1 / p)}\|\nabla u\|_{L^{p}(D)}^{q} \\
& \quad \leq c_{4}(n, p, q, D)\|\nabla u\|_{L^{p}(D)}^{q} .
\end{aligned}
$$

Condition (i) is proved.

The following example shows that the upper bound for $q$ in Theorem 3.4 is essentially sharp, and hence there are domains satisfying a quasihyperbolic boundary condition and which are not $(n p /(n-p), p)$-Poincaré domains for any $1 \leq p<n$. For simplicity we consider here the case $n=2$ : 
3.5. Example. Let $G_{0}$ be the open rectangle bounded by the lines

$$
x_{1}=0, \quad x_{2}=0, \quad x_{1}=1, \quad x_{2}=-1
$$

and for $j=1,2, \ldots$ let $G_{j}$ be the open triangle bounded by

$$
x_{1}=2^{-2 j}, \quad x_{2}=2^{-2 j}-2^{-2 b j}, \quad x_{1}+x_{2}=2^{-2 j}-2^{-2 b j},
$$

where $b \geq 2$ is a constant. Denote by $\hat{G}$ the reflection of the domain $\cup_{j=0}^{\infty} G_{j}$ with respect to the line $x_{2}=-\frac{1}{2}$. Set

$$
G=\bigcup_{j=1}^{\infty} G_{j} \cup \hat{G}
$$

Let $T: \mathbf{R}^{2} \rightarrow \mathbf{R}^{2}$ be a translation such that $T\left(x_{1}, x_{2}\right)=\left(x_{1}, x_{2}+\frac{1}{2}\right)$. Set $D=$ $T(G) . D$ satisfies a quasihyperbolic boundary condition with $a=36 b$.

Let $G_{j}^{1}$ be the open set bounded by the lines $x_{1}=2^{-2 j}, x_{2}=2^{-2 j}-2^{-2 b j}$, $x_{2}=2^{-2 b j}, x_{1}+x_{2}=2^{-2 j}-2^{-2 b j}$. Let $\hat{G}_{j}^{1}$ be the image of $G_{j}^{1}$ under reflection across the line $x_{2}=-\frac{1}{2}$. Set $T\left(G_{j}^{1}\right)=D_{j}^{1}$ and $T\left(\hat{G}_{j}^{1}\right)=\hat{D}_{j}^{1}$.

Choose a piecewise linear continuous function $u: D \rightarrow \mathbf{R}$ such that

$$
u(x)= \begin{cases}2^{4 j / q} & \text { in } D_{j}^{1}, j=1,2, \ldots \\ 0 & \text { in }\left\{\left(x_{1}, x_{2}\right) \mid x_{1} \in(0,1), x_{2} \in\left(-\frac{1}{2}, \frac{1}{2}\right)\right\} \\ -2^{4 j / q} & \text { in } \hat{D}_{j}^{1}, j=1,2, \ldots\end{cases}
$$

Now, $u_{D}=0$, and

$$
\int_{D}|u(x)|^{q} d x \geq \infty
$$

and

$$
\int_{D}|\nabla u(x)|^{p} d x=3 \sum_{j=1}^{\infty} 2^{(4 p / q+2 b p) j} 2^{-4 b j}<\infty \quad \text { if } \frac{2 p}{q}+b p-2 b<0 .
$$

So, if $q=2 p /(2-p)$ and $1 \leq p<2$, then $D \notin \mathscr{P}(2 p /(2-p), p)$ for any $1 \leq p<2$. In fact, $D$ is not a $(q, p)$-Poincaré domain for any $q>2 p / b(2-p)$, where $1 \leq p<2$.

3.6. A similar example of the well known 'rooms and passages' domain $D$ in $\mathbf{R}^{n}$ shows that $D$ is not an $(n p /(n-p), p)$-Poincaré domain for any $1 \leq p<n$. See $[H 1,5.9]$.

A domain $D$ in $\mathbf{R}^{n}$ is called starshaped with respect to a point $x_{0} \in D$ if there is a point $x_{0} \in D$ such that the set $\left\{x_{0}+\left(x-x_{0}\right) t \mid t \in[0,1]\right\} \subset D$ for each $x \in D$. Bounded domains starshaped with respect to a point are $(p, p)$-Poincaré domains for each $p \in[1, \infty)$, [H1, Theorem 3.1] and [SS1, Theorem 6]. 
Examples 3.7 and 4.3 show that there are domains starshaped with respect to a point which are not $(n p /(n-p), p)$-Poincaré domains.

3.7. Example. Let $D=D_{1} \cup D_{2}$ where

$$
\begin{aligned}
& D_{1}=\left\{\left(x_{1}, x_{2}\right)|| x_{2} \mid<x_{1}^{2}, 0<x_{1}<1\right\}, \\
& D_{2}=\left\{\left(x_{1}, x_{2}\right)|| x_{2} \mid<\left(x_{1}-2\right)^{2}, \quad 1 \leq x_{1}<2\right\} .
\end{aligned}
$$

$D$ is not a John domain but $D$ is starshaped with respect to $(1,0)$. Set

$$
T_{i}=\left\{\left(x_{1}, x_{2}\right) \in D_{1} \mid 2^{-i}<x_{1}<2^{-i+1}\right\},
$$

and let $\hat{T}_{i}$ be the reflection of $T_{i}$ with respect to the line $x_{1}=1$. Let $\left(u_{i}\right)_{i=2}^{\infty}$ be a sequence of piecewise linear continuous functions defined by

$$
u_{i}(x)= \begin{cases}2^{3 i / q}, & x \in T_{i} \\ 0, & x \in D \backslash \bigcup_{j=i-1}^{i+1}\left(T_{j} \cup \hat{T}_{j}\right) \\ -2^{3 i / q}, & x \in \hat{T}_{i} .\end{cases}
$$

Then

$$
\int_{D}\left|u_{i}(x)\right|^{q} d x \geq \int_{T_{i}}\left|u_{i}(x)\right|^{q} d x \geq \frac{7}{3}
$$

and

$$
\int_{D}\left|\nabla u_{i}(x)\right|^{p} d x \leq 4 \int_{T_{i}} 2^{(3 p / q+p) i} d x=\frac{448}{3} 2^{(-3+p+3 p / q) i} \rightarrow 0,
$$

if $i \rightarrow \infty$ and $-3+p+3 p / q<0$.

If $q=2 p /(2-p)$ and $1 \leq p<2$, then $D \notin \mathscr{P}(2 p /(2-p), p)$. More generally, if $D=D_{1} \cup D_{2}$, where

$$
\begin{aligned}
& D_{1}=\left\{\left(x_{1}, x_{2}\right)|| x_{2} \mid<x_{1}^{k}, x_{1} \in(0,1)\right\} \quad \text { and } \\
& D_{2}=\left\{\left(x_{1}, x_{2}\right)|| x_{2} \mid<1, x_{2} \in[1,2)\right\}, k>1,
\end{aligned}
$$

then $D \notin \mathscr{P}(q, p)$ for any $q>p /\left(1-p(k+1)^{-1}\right), 1 \leq p<2$.

\section{Unbounded Poincaré domains with infinite Lebesgue measure}

4.1. Theorem. Suppose that $D$ in $\mathbf{R}^{n}$ is an unbounded domain such that $D=\cup_{i=1}^{\infty} D_{i}$, where $D_{i} \in \mathscr{P}(n p /(n-p), p)$ with Poincaré constants $c\left(D_{i}\right) \leq c_{0}$ for some constant $c_{0}$, and $D_{i} \subset \bar{D}_{i} \subset D_{i+1}, i=1,2, \ldots$, and $\left|D_{1}\right|>0$. Then there is a constant $c_{1}<\infty$ such that

$$
\inf _{a \in R}\|u-a\|_{L^{n p /(n-p)}(D)} \leq c_{1}\|\nabla u\|_{L^{p}(D)}
$$

whenever $u \in L_{p}^{1}(D), 1 \leq p<n$. 
The idea for the proof is from [IN].

Proof. Let $u \in L_{p}^{1}(D)$. By [Maz, 1.1.2. Theorem] $u \in L_{p}(D$, loc $)$. Set

$$
u_{i}=\frac{1}{\left|D_{i}\right|} \int_{D_{i}} u(x) d x, \quad i=1,2, \ldots
$$

We will show that there is a convergent subsequence $\left(u_{i j}\right)$ of $\left(u_{i}\right)$ and $b \in \mathbf{R}$ such that $\lim _{j \rightarrow \infty} u_{i j}=b$ and

$$
\|u-b\|_{L^{n p /(n-p)}(D)} \leq c\left(p, D, c_{0}\right)\|\nabla u\|_{L^{p}(D)} .
$$

The triangle inequality yields

$$
\left|u_{i}\right|=\left|D_{1}\right| \int_{D_{1}}\left|u_{i}\right| d x \leq\left|D_{1}\right|\left(\int_{D_{1}}\left|u_{i}-u(x)\right| d x+\int_{D_{1}}|u(x)| d x\right)
$$

where

$$
\int_{D_{1}}|u(x) d x|<\infty, \quad \text { since } u \in L_{p}(D, \text { loc }) .
$$

Since $D_{i}$ is a $(n p /(n-p))$-Poincaré domain with a constant $c_{i}(D) \leq c_{0}$, we obtain

$$
\begin{aligned}
\int_{D_{1}} \mid u(x)- & \left.u_{i}|d x \leq| D_{1}\right|^{1-1 / p+1 / n}\left\|u-u_{i}\right\|_{L^{n p /(n-p)}\left(D_{1}\right)} \\
& \leq\left|D_{1}\right|^{1-1 / p-1 / n}\left\|u-u_{i}\right\|_{L^{n p /(n-p)}\left(D_{i}\right)} \leq c_{0}\left|D_{1}\right|^{1-1 / p+1 / n}\|\nabla u\|_{L^{p}(D)} \\
& <\infty .
\end{aligned}
$$

Thus $\left(u_{i}\right)$ is a bounded sequence and hence there is a convergent subsequence $\left(u_{i j}\right)$ and $b \in \mathbf{R}$ such that $\lim _{j \rightarrow \infty} u_{i j}=b$.

Since

$$
\lim _{j \rightarrow \infty} \chi_{D_{j}}(x)\left|u(x)-u_{j}\right|^{n p /(n-p)}=\chi_{D}(x)|u(x)-b|^{n p /(n-p)},
$$

Fatou's lemma and the fact that Poincaré constants of $D_{i}$ are less than $c_{0}$ yield

$$
\begin{aligned}
\int_{D}|u(x)-b|^{n p /(n-p)} d x=\int_{D} \liminf _{j \rightarrow \infty} \chi_{D_{j}}(x)\left|u(x)-u_{j}\right|^{n p /(n-p)} d x \\
\left.\quad \leq \liminf _{j \rightarrow \infty} \int_{D} \chi_{D_{j}}(x)\left|u(x)-u_{j}\right|^{n p /(n-p}\right) d x \\
\leq \liminf _{j \rightarrow \infty} c_{1}\left(n, p, c_{0}\right)\left(\left(\int_{D_{j}}|\nabla u(x)|^{p} d x\right)^{1 / p}\right)^{n p /(n-p)} \\
\leq \liminf _{j \rightarrow \infty} c_{1}\left(n, p, c_{0}\right)\|\nabla u\|_{L^{p}(D)}^{n p /(n-p)}=c_{1}(n, p)\|\nabla u\|_{L^{p}(D)}^{n p /(n-p)} .
\end{aligned}
$$

The inequality (4.2) is proved. 
4.3. Examples. (a) Theorem 4.1 yields that the following domains

1. $\mathbf{R}^{n}$

2. $\mathbf{R}^{n} \backslash\left\{\left(x_{1}, x_{2}, \ldots, x_{n}\right) \mid x_{2}=\ldots=x_{n}=0, x_{1}>0\right\}$

3. $\left\{\left(x^{\prime}, x_{n}\right) \in \mathbf{R}^{n}\left|x_{n}>\right| x^{\prime} \mid\right\}$

are $(n p /(n-p), p)$-Poincaré domains for each $1 \leq p<n$.

(b) The following domain $D$ is a $(2 p /(2-p), p)$-Poincaré domain, for all $p \in[1,2)$. The domain $D$ is due to J. Väisälä. Fix points $a_{2 k+1}=\left(\frac{1}{2} \pi,(2 k+1) !\right)$, $b_{2 k+1}=\left(\frac{7}{6} \pi,(2 k+1) !\right)$ and $c_{2 k+1}=\left(\frac{11}{6} \pi,(2 k+1) !\right)$ from the circles $\left\{x \in \mathbf{R}^{2} \mid\right.$ $|x|=(2 k+1) !\} ; k=0,1,2, \ldots$ Fix points $x_{2 k}=\left(\frac{1}{6} \pi, 2 k !\right), y_{2 k}=\left(\frac{5}{6} \pi, 2 k !\right)$ and $z_{2 k}=\left(\frac{3}{2} \pi, 2 k !\right)$ from the circles $\left\{x \in \mathbf{R}^{2}|| x \mid=2 k !\right\} ; k=1,2, \ldots$. Fix $c \geq 11$. Join $x_{2 i}$ to $y_{2 i}$ by a $c$-cigar $\operatorname{cig}(E, c)=A_{2 i+1}$ the core being $\left[x_{2 i}, a_{2 i+1}\right] \cup\left[a_{2 i+1}, y_{2 i}\right]$. Join $y_{2 i}$ to $z_{2 i}$ by a $c$-cigar $\operatorname{cig}(E, c)=B_{2 i+1}$ the core being $\left[y_{2 i}, b_{2 i+1}\right] \cup\left[b_{2 i+1}, z_{2 i}\right]$. Join $z_{2 i}$ to $x_{2 i}$ by a $c$-cigar $\operatorname{cig}(E, c)=C_{2 i+1}$ the core being $\left[z_{2 i}, c_{2 i+1}\right] \cup\left[c_{2 i+1}, x_{2 i}\right]$. Join $c_{2 i-1}$ to $a_{2 i-1}$ by a $c$-cigar $\operatorname{cig}(E, c)=$ $X_{2 i}$ the core being $\left[c_{2 i-1}, x_{2 i}\right] \cup\left[x_{2 i}, a_{2 i-1}\right]$. Join $a_{2 i-1}$ to $b_{2 i-1}$ by a $c$-cigar $\operatorname{cig}(E, c)=Y_{2 i}$ the core being $\left[a_{2 i-1}, y_{2 i}\right] \cup\left[y_{2 i}, b_{2 i-1}\right]$. Join $b_{2 i-1}$ to $c_{2 i-1} A$ by a $c$-cigar $\operatorname{cig}(E, c)=Z_{2 i}$ the core being $\left[b_{2 i-1}, z_{2 i}\right] \cup\left[z_{2 i}, c_{2 i-1}\right]$.

Define

$D_{1}=X_{2} \cup Y_{2} \cup Z_{2} \cup A_{3} \cup C_{3} \cup X_{4}$,

$D_{2}=D_{1} \cup B_{3} \cup Y_{4} \cup Z_{4} \cup A_{5} \cup C_{5} \cup X_{6}$,

$D_{3}=D_{2} \cup B_{5} \cup Y_{6} \cup Z_{6} \cup A_{7} \cup C_{7} \cup X_{8}$,

$D_{i}=D_{i-1} \cup B_{2 i-1} \cup Y_{2 i} \cup Z_{2 i} \cup A_{2 i+1} \cup C_{2 i+1} \cup X_{2 i+1}, \ldots$

Domains $D_{i}$ can be constructed so that $D_{i}$ is $2 c$-John domain for each $i=$ $1,2, \ldots$, and hence

$$
D=\bigcup_{i=1}^{\infty} D_{i}
$$

satisfies (4.2) whenever $1 \leq p<2=n$ and $u \in L_{p}^{1}(D) \cup L^{1}\left(D_{i}\right)$ by Theorem 3.1 and proof for Theorem 4.1.

(c) The following domains are not $(n p /(n-p), p)$-Poincaré domains for any $1 \leq p<n$ :

1. $D_{1}=\left\{\left(x_{1}, \ldots, x_{n}\right) \in \mathbf{R}^{n}|| x_{i} \mid<1, i=1, \ldots, n-1\right\}$. For example, define a continuous piecewise linear function $u$ on $D_{1}$ such that $u(x)=1$, for $x_{n}>2$, and $u(x)=0$, for $0<x_{n}<1$ and $u$ is odd in $x_{n}$. Then $u \in L_{p}^{1}\left(D_{1}\right)$ and $\int_{D_{1}}|\nabla u(x)|^{p} d x \leq 2^{n}$ and $\int_{D_{1}}|u(x)|^{q} d x=\infty, q=n p /(n-p)$.

2. $D_{2}=\left\{\left(x^{\prime}, x_{n}\right) \in \mathbf{R}^{n}|| x^{\prime}\left|-1<x_{n}<1-\right| x^{\prime} \mid\right\}$, ([Maz, 4.7.4. Example]).

3. Let $D=G \cup\left\{\left(x_{1}, x_{2}\right) \in \mathbf{R}^{2} \mid x_{1}>\frac{1}{2}\right.$ and $\left.\left|x_{2}\right|<x_{1}\right\}$, where $G$ is the domain from Example 3.5. Then $D$ satisfies a quasihyperbolic boundary condition, $|D|=\infty$, and $D \notin \mathscr{P}(n p /(n-p), p)$ for any $1 \leq p<2$. 
4. Let $D=\left\{\left(x_{1}, x_{2}\right) \in \mathbf{R}^{2}|| x_{2} \mid<x_{1}^{2}\right\}$. Now $D$ is starshaped with respect to $(1,0)$, and $D \notin \mathscr{P}(2 p /(2-p), p)$ for any $1 \leq p<2$, see Example 3.7.

4.4. Unbounded John domains. The concept 'a John domain' has been extended for unbounded domains in [NV], [V1]. Let $E$ be a closed arc with endpoints $a$ and $b$. The subarc between $x$ and $y$ is denoted by $E[x, y]$. For $x$ in $E \backslash\{a, b\}$ write

$$
q(x)=\min \{\operatorname{dia}(E[a, x]), \operatorname{dia}(E[b, x])\} .
$$

Let $c \geq 1$. A domain $D$ in $\mathbf{R}^{n}$ is a $c$-John domain, if each pair of distinct points $a$ and $b$ in $D$ can be joined by an arc $E$ such that

$$
\operatorname{cig} E(a, b)=\bigcup\left\{B\left(x, \frac{q(x)}{c}\right) \mid x \in E \backslash\{a, b\}\right\} \subset D .
$$

The set $\operatorname{cig} E(a, b)$ is called a $c$-cigar with core $E$ joining $a$ and $b$.

Whenever $D$ is bounded this gives exactly an $(\alpha, \beta)$-John domain for some $\alpha$ and $\beta$.

The following result due to J. Väisälä, [V2], makes it possible to resolve the Poincaré domain question for unbounded John domains.

4.5. Theorem [V2]. Let $D$ be an unbounded $c$-John domain. There are bounded $c^{\prime}$-John domains $D_{i}$ such that $D_{i} \subset \bar{D}_{i} \subset D_{i+1}, i=1,2, \ldots$ and $D=\cup_{i=1}^{\infty} D_{i}$.

I would like to thank J. Väisälä for his interest in this problem and his time invested in Theorem 4.5.

Theorems 3.1, 4.1 and 4.5 imply the main result of this chapter:

4.6. Corollary. An unbounded $c$-John domain $D$ in $\mathbf{R}^{n}$ is a $(n p /(n-p), p)$ Poincaré domain, $1 \leq p<n$.

4.7. Remarks. (1) V.G. Maz'ya has given a necessary and sufficient condition for $D$ to satisfy the inequality

$$
\|u-a\|_{L^{n p /(n-p)}(D)} \leq c\|\nabla u\|_{L^{p}(D)}
$$

where $u \in L_{p}^{1}(D), 1 \leq p<n$, and $c$ is independent of the function $u$ and $a=\inf \{t:|\{x: u(x)>t\}|<\infty\},[\mathrm{Maz}, 4.7 .4$. Theorem]. The number $a<\infty$, [Maz, 4.7.4. Lemma 2].

(2) Suppose that $A_{i} \subset \mathbf{R}^{n}, i=1,2$, are $(n p /(n-p), p)$-Poincaré domains such that $\left|A_{1}\right|<\infty$, and $\left|A_{2}\right|=\infty$, and $\left|A_{1} \cap A_{2}\right|>0$. Then $A_{1} \cup A_{2} \in$ 
$\mathscr{P}(n p /(n-p), p),[\mathrm{A}]$. If both sets $A_{i}, i=1,2$, are unbounded $(n p /(n-p), p)$ Poincaré domains with infinite measure, then the union $A_{1} \cup A_{2}$ is not necessarily in $\mathscr{P}(n p /(n-p), p)$. See Example 4.4(b)(2), where

$$
\begin{aligned}
& A_{1}=\left\{\left(x^{\prime}, x_{n}\right) \in \mathbf{R}^{n}\left|x_{n}>\right| x^{\prime} \mid-1\right\} \quad \text { and } \\
& A_{2}=\left\{\left(x^{\prime}, x_{n}\right) \in \mathbf{R}^{n}\left|x_{n}<1-\right| x^{\prime} \mid\right\} .
\end{aligned}
$$

(3) Locally $L$-bilipschitz homeomorphisms preserve $(q, p)$-Poincaré domains, also when a domain is unbounded and $q=n p /(n-p)$. See [H1, Theorem 2.1].

\section{Weighted Poincaré inequalities}

By a weight we mean a non-negative measurable function on $\mathbf{R}^{n}$. $Q \subset \mathbf{R}^{n}$

A weight $w$ is said to satisfy the $A_{p, q}$-condition, $w \in A_{p, q}$, if for each cube

$$
\left(\frac{1}{|Q|} \int_{Q} w(x)^{q} d x\right)^{1 / q}\left(\frac{1}{|Q|} \int_{Q} w(x)^{-p /(p-1)}\right)^{(p-1) / p} \leq c
$$

$1<p<\infty, 1<q<\infty$. The infimum over the $c$ 's is called the $A_{p, q}$-constant of $w$ and is denoted by $a_{p, q},[\mathrm{MW}]$.

For example $w(x)=1$ and $w(x)=|x|^{\alpha}, \alpha<n(1-(1 / p))$, are $A_{p, q}$-weights, $q=n p /(n-p(n-\lambda)), 0<\lambda<n$, and especially $w(x)=|x|^{\alpha}, \alpha<n(1-(1 / p))$ is an $A_{p, n p /(n-p)}$-weight, [SW, Theorem B*] and [MW, Theorem 4].

Recall that a weight $w$ is a Muckenhoupt weight, $w \in A_{p}, 1<p<\infty$, if for each cube $Q \subset \mathbf{R}^{n}$

$$
\left(\frac{1}{|Q|} \int_{Q} w(x) d x\right)\left(\frac{1}{|Q|} \int_{Q} w(x)^{-1 /(p-1)} d x\right)^{p-1} \leq c
$$

If $w \in A_{p, q}$, then $w^{q} \in A_{r}$, where $r=1+q p /(p-1)$. For an $(\alpha, \beta)$-John domain we obtain

5.1. Theorem. Suppose that $D \in J(\alpha, \beta)$ and $w \in A_{p, q}$, where $q=$ $n p /(n-p), 1<p<n$. Then there is a constant $c=c\left(n, p, a_{p, q}\right)$ such that

$$
\left\|u-u_{D, w}\right\|_{L^{q}(D, w)} \leq c\left(\frac{\beta}{\alpha}\right)^{16 n}\|\nabla u\|_{L^{p}(D, w)}
$$

whenever $u \in C^{\infty}(D)$. 
Proof. Let $u \in C^{\infty}(D)$. Extend $u$ as 0 into $\mathbf{R}^{n} \backslash D$. By [M, Lemma 3.3] and [MW, Theorem 4]

$$
\begin{aligned}
& \left(\int_{D}\left|u(x)-u_{E}\right|^{q} w(x)^{q} d x\right)^{1 / q} \\
& \quad \leq c_{1}(n)\left(\frac{\beta}{\alpha}\right)^{16 n}\left(\int_{D}\left(\int_{D}|x-y|^{1-n}|\nabla u(y)| d y\right)^{q} w(x)^{q} d x\right)^{1 / q} \\
& \quad \leq c_{2}\left(n, p, a_{p, q}\right)\left(\frac{\beta}{\alpha}\right)^{16 n}\left(\int_{D}|\nabla u(x)|^{p} w(x)^{p} d x\right)^{1 / p}
\end{aligned}
$$

where $E=B^{n}\left(x_{0}, c(n)\left(\alpha^{5}\right) /\left(\beta^{4}\right)\right)$ and $x_{0}$ is a John center.

A lemma similar to [H2, Lemma 2.2] implies the required inequality.

As in the case without weights Theorem 5.1 yields:

5.2. Theorem. Suppose that $D$ is an unbounded $c_{0}$-John domain in $\mathbf{R}^{n}$. Let $w \in A_{p, q}$, where $q=n p /(n-p)$, and $1<p<n$. Then there is a constant $c_{1}=c_{1}\left(n, p, a_{p, q}, c_{0}\right)<\infty$ such that

$$
\inf _{a \in \mathbf{R}}\|u-a\|_{L^{n p /(n-p)}(D, w)} \leq c_{1}\|\nabla u\|_{L^{p}(D, w)}
$$

whenever $u \in C^{\infty}(D)$.

5.3. Remark. E. Fabes, C. Kenig and R. Serapioni have proved the weighted $(k p, p)$-Poincaré inequality for a cube $Q$ in $\mathbf{R}^{n}$ :

$$
\left(\int_{Q}\left|u(x)-u_{Q, w}\right|^{k p} w(x) d x\right)^{1 / k p} \leq c\left(\int_{Q}|\nabla u(x)|^{p} w(x) d x\right)^{1 / p},
$$

where $c=c(k, n, p)|Q|^{1 / n} w(Q)^{1 / k p-1 / p}, w \in A_{p}, 1<p<\infty$, a positive number $k$ can be slightly bigger than one, and $c$ is a constant independent of $u \in C^{\infty}(\bar{Q})$, [FKS].

This result can be generalized to an $(\alpha, \beta)$-John domain in $\mathbf{R}^{n}$ using the integral representation from [M, Lemma 3.3] and the technique from [FKS].

\section{References}

[A] Andersson, R.: Unbounded Soboleff regions. - Math. Scand. 13, 1963, 75-89.

[B] Bojarski, B.: Remarks on Sobolev imbedding inequalities. - Proceedings of the conference Complex Analysis, Joensuu, 1987. Lecture Notes in Mathematics 1351, SpringerVerlag, 1988, 52-68.

[EO] Edmunds, D.E., and B. OpIC: Weighted Poincaré and Friedrichs inequalities. - To appear.

[EH] Evans, W.D., and D.J. HaRris: Sobolev embeddings for generalized ridged domains. Proc. London Math. Soc. (3) 54, 1987, 141-175. 
[FKS] Fabes, E., C. Kenig, and R. Serapioni: The local regularity of solutions of degenerate elliptic equations. - Comm. Partial Differential Equations 7, 1982, 77-116.

[GHM] Gehring, F.W., K. HAG, and O. Martio: Quasihyperbolic geodesics in John domains. - Math. Scand. 65, 1989, 75-92.

[GM] Gehring, F.W., and O. Martio: Lipschitz classes and quasiconformal mappings. - Ann. Acad. Sci. Fenn. Ser. A I Math. 10, 1985, 203-219.

[GP] Gehring, F.W., and B.P. Palka: Quasiconformally homogeneous domains. - J. Analyse Math. 30, 1976, 172-199.

[GO] Gurka, P., and B. OpIC: $N$-dimensional Hardy inequality and imbedding theorems for weighted Sobolev spaces on unbounded domains. - Proceedings of the International Summer School on Function Spaces, Differential Operators and Nonlinear Analysis held in Sodankylä. Longman Scientific and Technical Publishers, 1989, 108-124.

[H1] HURRI, R.: Poincaré domains in $\mathbf{R}^{n}$. - Ann. Acad. Sci. Fenn. Ser. A I Math. Dissertationes $71,1988,1-41$.

[H2] HurRI, R.: The weighted Poincaré inequalities. - Math. Scand. 67, 1990, 145-160.

[IN] IWANIEC, T., and C.A. NoldER: Hardy-Littlewood inequality for quasiregular mappings in certain domains in $\mathbf{R}^{n}$. - Ann. Acad. Sci. Fenn. Ser. A I Math. 10, 1985, 267-282.

[K] Kufner, A.: Weighted Sobolev spaces. - John Wiley \& Sons, 1985.

[M] Martio, O.: John domains, bilipschitz balls and Poincaré inequality. - Rev. Roumaine Math. Pures Appl. 33:1-2, 1988, 107-112.

[MS] Martio, O., and J. Sarvas: Injectivity theorems in plane and space. - Ann. Acad. Sci. Fenn. Ser. A I Math. 4, 1978-79, 383-401.

[MV] Martio, O., and M. Vuorinen: Whitney cubes, p-capacity, and Minkowski content. Exposition. Math. 5, 1987, 17-40.

[MW] Muckenhoupt, B., and B.L. Wheeden: Weighted norm inequalities for fractional integrals. - Trans. Amer. Math. Soc. 192, 1974, 261-274.

[Maz] Maz'YA, V.G.: Sobolev spaces. - Springer-Verlag, 1985.

[NV] NÄKKI, R., and J. VÄISÄLÄ: John disks. - Exposition. Math. 9, 1991, 3-43.

[SS1] Sмith, W., and D. Stegenga: Hölder domains and Poincaré domains. - Trans. Amer. Math. Soc. 319, 1990, 67-100.

[SS2] Smith, W., and D. Stegenga: Sobolev imbeddings and integrability of harmonic functions on Hölder domains. - To appear.

[SS3] Smith, W., and D. Stegenga: Exponential integrability of the quasi-hyperbolic metric on Hölder domains. - Ann. Acad. Sci. Fenn. Ser. A I Math. 16, 1991, 344-359.

[S] Stein, E.M.: Singular integrals and differentiability properties of functions. - Princeton Univ. Press, Princeton, N.J., 1970.

[SW] Stein, E., and G. WeIss: Fractional integrals on $n$-dimensional Euclidean space. - J. Math. Mech. 7, 1958, 503-514.

[V1] VÄIsÄLÄ, J.: Quasiconformal maps of cylindrical domains. - Acta Math. 162, 1989, 201225.

[V2] VÄISÄLÄ, J.: Exhaustions of John domains. - Unpublished manuscript. 